\title{
PARAMETERS SELECTION FOR ELECTROPOLISHING PROCESS OF PRODUCTS MADE OF COPPER AND ITS ALLOYS
}

\begin{abstract}
Electropolishing is electrochemical method used in metal working that has a vital role in production of medical apparatus, in food or electric industry. The purpose of this paper is to determine optimal current parameters and time required for conducting electropolishing process from the perspective of changes of surface microgeometry. Furthermore, effect of different types of mechanical working used before electropolishing on final surface state was evaluated by observation in changes of topography. Research was conducted on electrolytic copper and brass. Analysis of surface geometry and its parameters (Ra, Sa) was used as criterion describing efficiency of chemical electropolishing. Results of the experiment allow for current parameter optimization of electrochemical polishing process for selected non-ferrous alloys with preliminary mechanical preparation of the surface.

Keywords: electropolishing, copper, brass, surface roughness
\end{abstract}

\section{Introduction}

Polishing is basic method of surface finish of metal products aimed at obtaining required roughness and polish. In industry, as well as in craftwork, costs of mechanical polishing represent over $50 \%$ costs of finishing the elements [1,2]. Abrasive polishing using different type of disks covered with abrasive compound generates loss in material up to several percentage of mass in case of small diameter objects [3]. Metal recovery from polishing dust is inconvenient and expensive. In case of products of complex geometry and small dimensions, proposed method is electrochemical polishing. It allows for substantial treatment time and cost reduction. Moreover, it is possible to recover dissolved metals from electrolytes used during the process.

During electropolishing process, objects made with metal, as anode, are immersed in bath. With particular current and temperature conditions, roughness of the surface is dissolved resulting with smoothing and polishing. Description of electropolishing was presented in 1935 - 1936 by Jacquet, that was conducting research related to copper polishing in orthophosphate acid solution [4]. He noted dependency between potential and density of current on anode. As indicated in Fig. 1, distance between roughness vertices and surface ending are smaller than between recesses and surface ending. Electrical resistance by vertices is lower than by recesses. This reflects in current intensity being higher on vertices resulting in selective dissolving, that leads to polishing of microroughness of surface.



Fig. 1. Diffusion layer distribution during electropolishing [4]; $\alpha$ - distance between vertex and surface ending, $\beta$ - distance between recess and surface ending

Electropolishing can be preliminary or finishing treatment for other processes such as electrolytic coating. In case of finishing process, homogenous and pure surface is obtained with no side effects of mechanical polishing such as thermal stresses or particles stucked in metal surface. Furthermore, obtained surface characterizes with specific properties such as high light reflection coefficient, required in aluminum mirrors production and decorative finishing, additionally low friction factor required in gear wheel production [5]. Moreover, corrosion resistance of the electropolished surface increases, that is used for finishing water and steam apparatus, springs etc. [6]. Electropolishing used between operations allows for distinguishing of latent defects of material, distributed on the surface. Therefore, electropolishing between operations is used in surface scratch detection also connecting rod fractures, blade turbines etc. Electropolished surfaces used for electrolytic coating characterizes with increased adhesion. Due to microroughness reduction, actual coated surface 
is decreased, which increases effective current density $[7,8]$. In medical industry, electropolishing is used for medical equipment cleaning, it can also be used as preliminary processing before anodic treatment of implants, that improves substantially process of osteointegration [9].

Electropolishing has wide range of applications specifically as finishing processing of copper and its alloys manufacturing. Copper and brass elements are used for fittings, screw propellers, builders hardware, parts for pipes, water-sewerage systems, heating systems, cable clamps, terminal strips, parts of electrotechnics, matrices for typography production, music instruments, ammunition, jewellery and many others. Due to that, electropolishing is the key process of surface preparation $[10,11]$.

In this paper, experiment results aimed for determination of optimum current and time parameters, used for conduction of electropolishing process from perspective of changes of microgeometry of surface are presented. Additionally, an effect of various mechanical treatment used before electropolishing on final surface results is evaluated by changes in topography.

\section{Experimental method and results}

Experiment described in this paper was divided into two stages: first one represents selection of optimum parameters of electropolishing (time, current density); second describes an evaluation of influence of surface mechanical preparation before electropolishing on surface geometry after the process. Experiment was carried out on two metals: electrolytic copper of purity 99,99\% and brass M80 (CuZn20). Both materials derive from products after plastic working. From copper sheet, two discs were cut of $60 \mathrm{~mm}$ diameter. From brass flat bar, rectangular samples of $4.5 \times 6.5 \mathrm{~mm}$ dimensions. The aim was to obtain approximate areas in both types of samples that was $5660 \mathrm{~mm}^{2}$. In order to develop surface of samples before electropolishing, they were submitted to preliminary processing of grinding on abrasive papers of different grain sizes (320 and 500), sandblasting and brushing. Summary of samples manufacturing as well as denotation and surface preparation methods is presented in Table 1. Electropolishing process was conducted in tetraoxophosphoric (V) acid $\mathrm{H}_{3} \mathrm{PO}_{4}$ and chromium trioxide $\mathrm{CrO}_{3}$ solution. Scheme of the process is presented in Fig. 2.

TABLE 1

Denotation of samples used during research

\begin{tabular}{|c|c|}
\hline \hline $\begin{array}{c}\text { Sample } \\
\text { denotatnion }\end{array}$ & Surface preparation \\
\hline Cu I & Mechanical polishing with abrasive paper 320 \\
\hline Cu II & Mechanical polishing with abrasive paper 500 \\
\hline Cu III & Brushing with steel brush for 30 s \\
\hline Cu IV & Sandblasting with SiC 50mm for 30s \\
\hline CuZn I & Mechanical polishing with abrasive paper 320 \\
\hline CuZn II & Mechanical polishing with abrasive paper 500 \\
\hline CuZn III & Brushing with steel brush for 30 s \\
\hline CuZn IV & Sandblasting with SiC 50mm for 30s \\
\hline
\end{tabular}

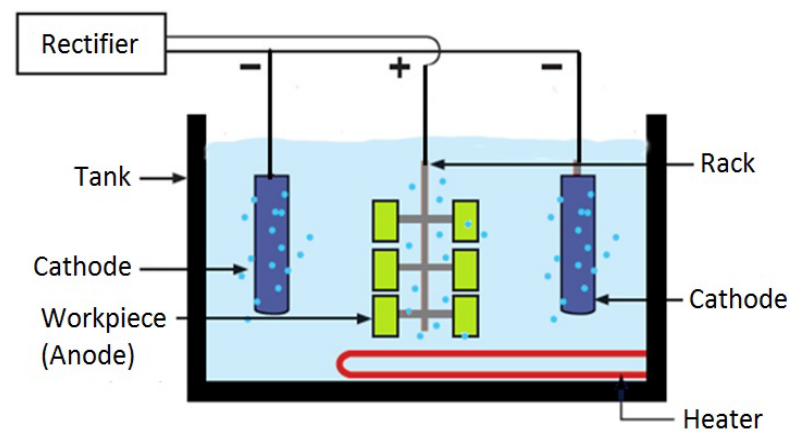

Fig. 2. Scheme of electropolishing process

For quantitative and qualitative verification in topography of surface microgeometry of samples, research was conducted using optical profilometer FRT Mikroprof CWL5000. Amongst available parameters of quantitative evaluation of roughness, $\mathrm{Ra}$ was selected, that is average arithmetical deviation from average line of the profile and $\mathrm{Sa}$ - average arithmetical roughness deviation determined in 3D system.

During first stage of an experiment, copper and brass samples were used after preliminary grinding with abrasive paper 320 (Cu I and CuZn I). Sample Cu I was submitted to electropolishing with the same current density $\left(14 \mathrm{~A} / \mathrm{dm}^{2}\right)$ and voltage $(8 \mathrm{~V})$ but with different process time. Results from profilometric measurements of roughness are presented in Fig. 3. For process efficiency evaluation, change in roughness referring to surface grinded with paper 320 was determined. Distinct decrease by half of roughness was observed after polishing for 10 minutes, which was defined as optimum. In Fig. 4 change in topography of sample surface $\mathrm{Cu}$ I before and after 10 minutes electropolishing process is shown.

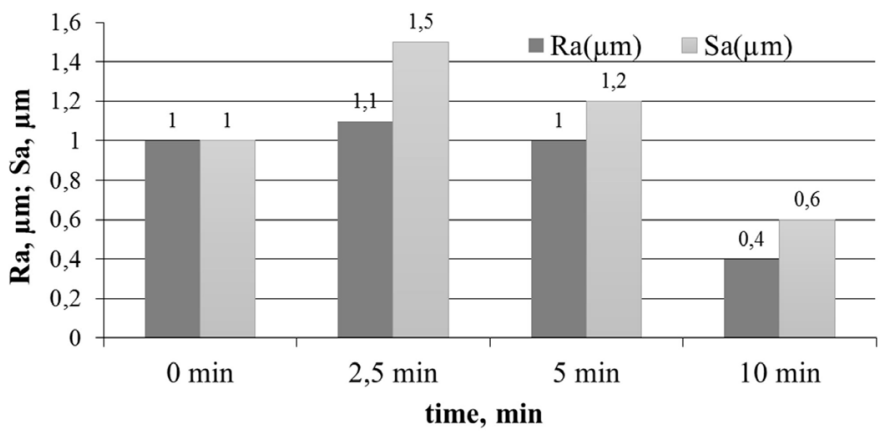

Fig. 3. Surface roughness parameters obtained after different electropolishing times of sample $\mathrm{Cu} \mathrm{I}$

In order to determine optimum current density in electropolishing process of copper and its alloys, research was done using the same time (10 minutes) and voltage $(8 \mathrm{~V})$ but different current density $\left(10,14\right.$ and $\left.17 \mathrm{~A} / \mathrm{dm}^{2}\right)$. Copper and brass samples were grinded with abrasive paper 320 (Cu I and $\mathrm{CuZn} \mathrm{I).} \mathrm{Obtained}$ results of surface roughness described with $\mathrm{Ra}$ and Sa parameters are presented in Fig. 5.

Second stage of conducted research was about determination how surface preparation influences an efficiency of 
a)
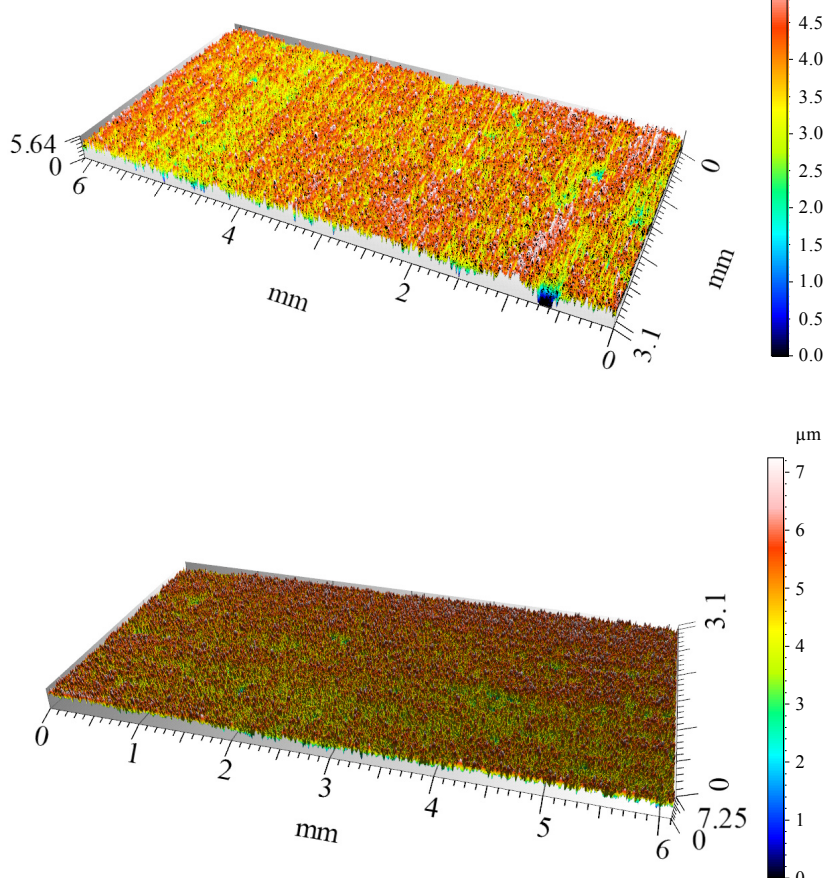

b)

Fig. 4. 3D surface topography of sample $\mathrm{Cu}$ I: a) initial state after grinding with abrasive paper 320; b) after electropolishing during 10 minutes
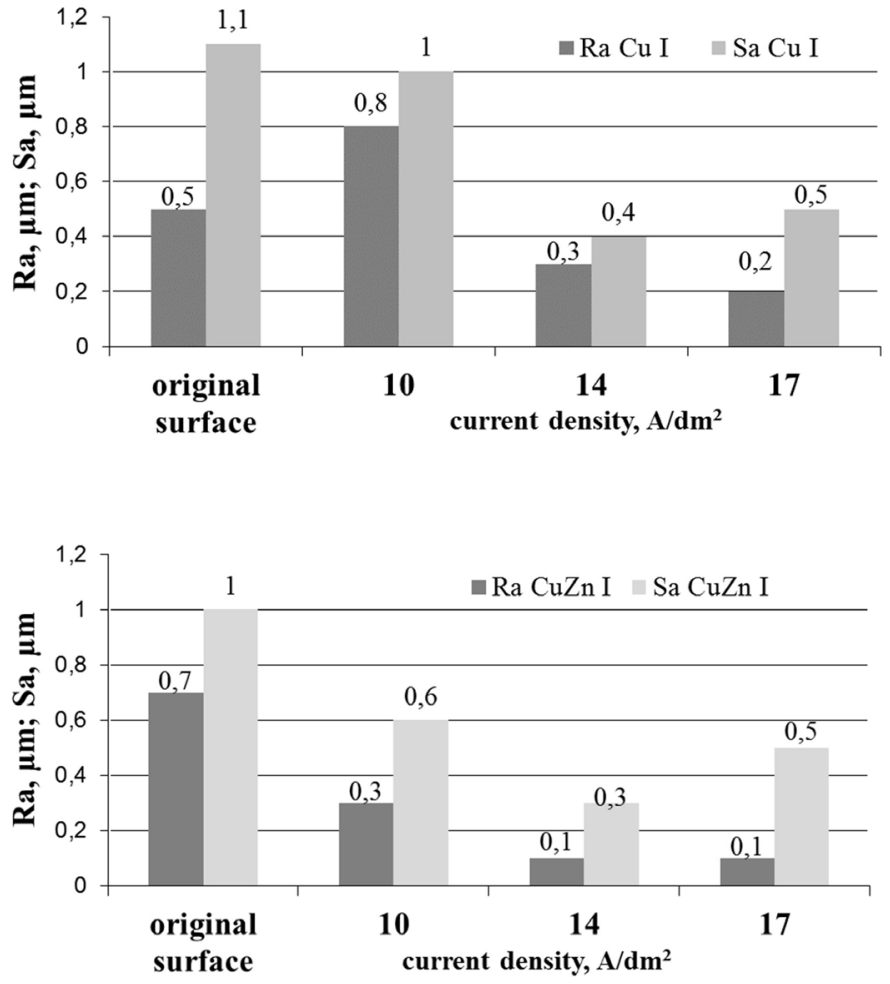

Fig. 5. Roughness parameters Ra and Sa with refer to current density of electropolishing for copper and brass surface grinded with abrasive paper 320

\section{Discussion}

Results obtained from conducted experiment allow for indicating dependence between electropolishing process and rate of surface geometry change of examined materials. Evaluation was done based on profile measurements, comparing typical roughness parameters which is $\mathrm{Ra}$ and $\mathrm{Sa}$. With increase of in Fig. 6.

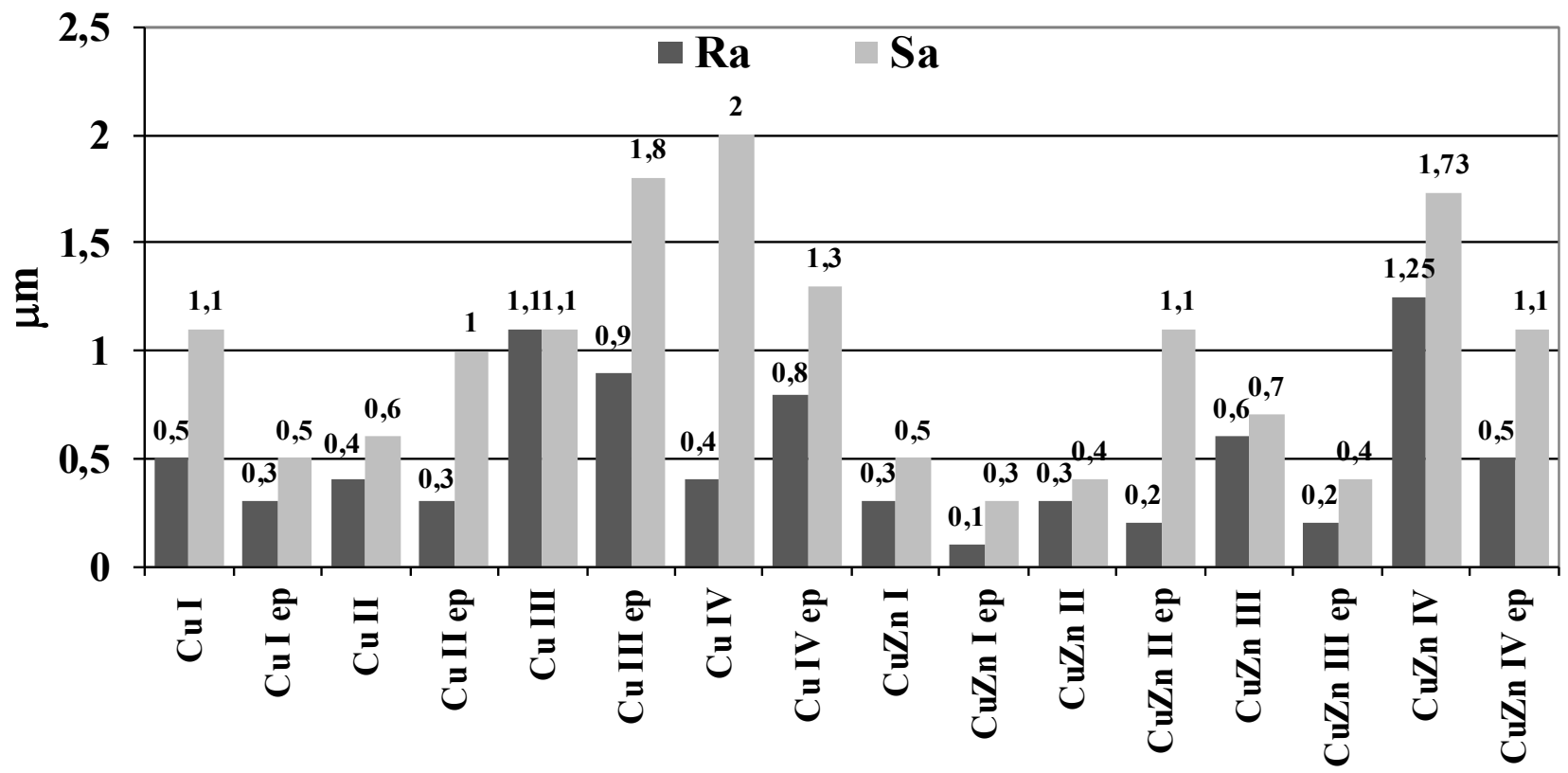

Fig. 6. Comparison of roughness parameters depending on surface preparation before electropolishing. Index „ep” indicates samples after electropolishing 
electropolishing time from 2,5 minute to 10 minutes, surface roughness decreases. In tested range of electropolishing timings, approx. halved roughness was obtained during 10 minutes of electropolishing (Fig. 3). It is also in line with 3D surface picture from Fig. 4. Similarly in case of evaluation of current density of electropolishing, in tested range from 10 to $17 \mathrm{~A} / \mathrm{dm}^{2}$, with an increase of current density, roughness of the surface was decreasing (Fig. 5).

Qualitative evaluation of changes in surface topography of samples after electropolishing was performed based on 3D view obtained in profilometric measurements. Pictures enable to visualize changes in topography during electropolishing. An example of those changes, mostly related to removal ridgings and scratches present after mechanical treatment is shown on Fig. 7.

a)
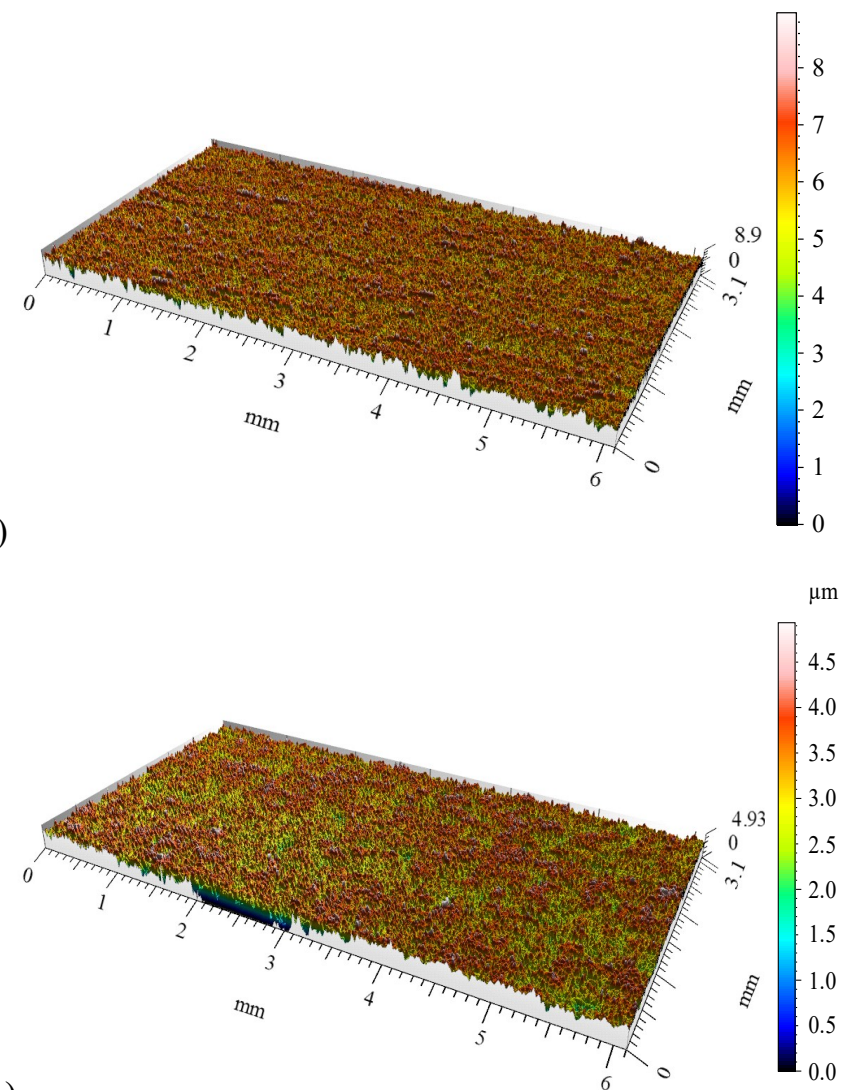

b)

Fig. 7. Picture of 3D surface topography of brass sample: a) initial state after brushing (CuZn III); b) after electropolishing for 10 minutes, current density $14 \mathrm{~A} / \mathrm{dm} 2$, voltage $8 \mathrm{~V}$ (CuZn III ep)

In parallel it was noted that with an increase of current density, a risk of local etching of polished surface increased, that was discovered in 3D view of electropolished brass after initial grinding with abrasive paper 500 (Fig. 8). It indicates that more beneficial from surface quality point of view is lengthening of polishing time with keeping possible low current density.

Results of surface measurements submitted to various preliminary treatment indicate that in agreed conditions of electropolishing, application of sandblasting results with lowest performance. In this case, there is no distinct roughness $\mu \mathrm{m}$

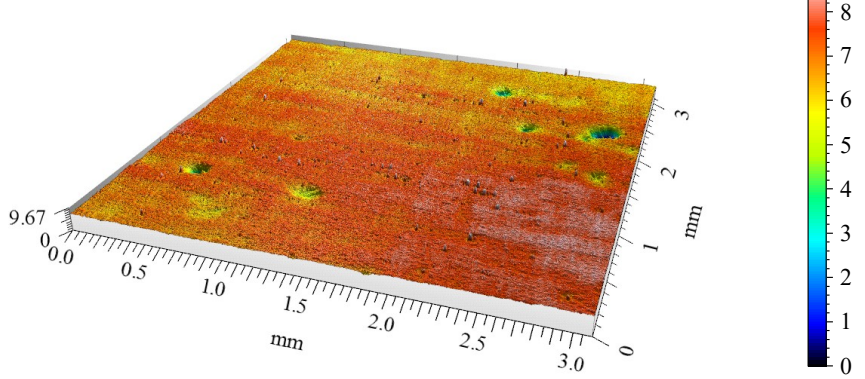

Fig. 8. 3D view of brass surface after electropolishing (CuZn II ep) with visible effects of local etching

decrease, which results with various consequences related to operational use. In case of sandblasting, it is crucial to select proper electropolishing parameters, enabling for obtaining results comparable with grinding and brushing (Fig. 6).

Electropolishing influences not only roughness $\mathrm{Ra}$, but also surface morphology by smoothening and reducing surface development in microscale. Fig. 9 presents an effect of changes in morphology of brass surface grinded with abrasive paper 500 (CuZn II). Comparing profiles from roughness distribution on sample surface before and after electropolishing it can be identified distinct decrease in height of roughness vertices from 5 to $2 \mu \mathrm{m}$. Rate of surface development described with shape and vertices quantity is also decreased.

a)

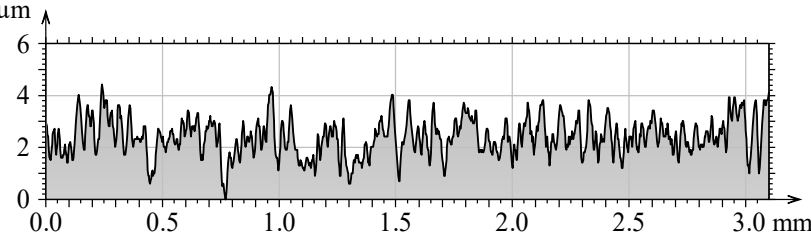

b)

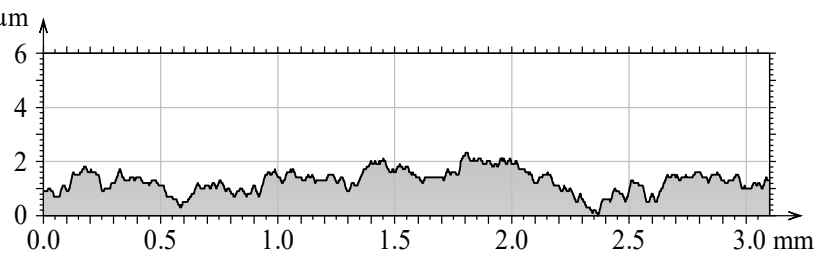

Fig. 9. Distribution of roughness on surface profile of sample CuZn II: a) surface grinded; b) surface grinded after electropolishing (10 min, $14 \mathrm{~A}, 8 \mathrm{~V}$ )

\section{Conclusion}

1. Based on conducted research optimal time of 10 minutes for electropolishing of copper and brass was identified.

2. For obtaining biggest reduction in roughness measured with $\mathrm{Ra}$ and Sa parameters, it is advised to use current density of $14 \mathrm{~A} / \mathrm{dm}^{2}$ and voltage $8 \mathrm{~V}$.

3. Profile measurement of surface before and after electropolishing allows for quantitative and qualitative evaluation 
of topography changes on the surface that can become effective tool in process of selection and verification of parameters for electropolishing.

4. Extending time of electropolishing can lead to local etching effect, which is indicated by an increase of Sa parameter.

\section{Acknowledgements}

This work was supported by Polish Ministry for Science and Higher Education under internal grant BK264/RM2/2016 for Institute of Metals Technology, Silesian University of Technology, Poland.

\section{REFERENCES}

[1] G. Nawrat, Electrochemical methods of surface engineering, 2010 Publishers Silesian University of Technology, Gliwice.
[2] S. Zaborski, A. Sudzik, T. Stechnij, Engineering Machinery 16, 117-124 (2011).

[3] S. Zaborski, A. Sudzik, D. Poroś, Mechanik 8, 356-368 (2015).

[4] Multi-author work, Electroplating handbook, 1985 WNT, Warszawa.

[5] A. Posmyk, The surface layers of aluminum construction materials, 2010 Publishers Silesian University of Technology, Gliwice.

[6] A. Kosmac, Electropolishing of stainless steel, Materials and applications, volume 11 Euro Inox, Brussels 2009.

[7] M.G. Fouad, F.N. Zein, M.I. Ismail, Electrochimica Acta 16, 1477-1487 (1971).

[8] D.R. Gabe, Corrosion Science 12, 113-120 (1972).

[9] P. Lochyński, B. Szczygieł, Chemik, 66, 3, 235-240 (2012).

[10] W. Tang et al., Physical Chemistry Chemical Physics 14, 76-81 (2012).

[11] A.M. Awada, N.A. Abdel Ghanyb, T.M. Dahyc, Applied Surface Science 256, 13, 4370-4375 (2010). 